\title{
Analysis of Hip Geometry by Clinical CT for the Assessment of Hip Fracture Risk in Elderly Japanese Women
}

\author{
Masako Ito ${ }^{1}$, Norimitsu Wakao ${ }^{2,4}$, Tetsuro $\mathrm{Hida}^{2}$, Yasumoto Matsui ${ }^{2}$, Yasue Abe ${ }^{3}$, \\ Kiyoshi Aoyagi ${ }^{3}$, Masataka Uetani ${ }^{1}$, Atsushi Harada ${ }^{2}$ \\ ${ }^{1}$ Department of Radiology, Nagasaki University School of Medicine \\ ${ }^{2}$ Department of Orthopaedic Surgery, National Center for Geriatrics and Gerontology \\ ${ }^{3}$ Department of Public Health, Nagasaki University School of Medicine \\ ${ }^{4}$ Department of Orthopaedic Surgery, Nagoya University School of Medicine
}

Corresponding Author:

Masako Ito

Department of Radiology, Nagasaki University School of Medicine

1-7-1 Sakamoto, Nagasaki 852-8501, JAPAN

phone : 81-95-819-7434

FAX : 81-95-819-7357

e-mail : masako@nagasaki-u.ac.jp 


\section{ABSTRACT}

Two case-control studies were designed to investigate the contribution of the geometry and bone mineral density (BMD) of the proximal femur to bone strength in Japanese elderly women. We also investigated whether clinical CT is useful to assess the risk of hip fracture. Subjects in the neck fracture study included 20 Japanese women with neck fracture (age: mean $\pm S D$; $80.1 \pm 4.5$ years old) and 20 age-matched control women (79.2 \pm 2.6 years old). Subjects in the trochanteric fracture study included 16 Japanese women with trochanteric fracture (82.6 \pm 5.0 years old) and 16 age-matched control women (80.8 \pm 3.8 years old). CT examination of the proximal femur was performed between the date of admission and the date of surgery. The CT scanners used were an Aquillion 16 (Toshiba) and Somatom 64 (Siemens); the scanning conditions including spatial resolution and scanning energy were adjusted, and the same type of reference phantom containing hydroxyapatite was used. QCT PRO software (Mindways, USA) was used to analyze data for BMD, geometry, and biomechanical parameters. Both the neck and trochanteric fracture cases had significantly lower total and cortical BMD, a significantly smaller cortical cross-sectional area (CSA) and a larger trabecular CSA. Both had significantly thinner cortex and smaller distance to center of bone mass, and women with trochanteric fracture had a significantly smaller cortical perimeter in the cross-sectional femoral neck. Women with neck fracture had a longer hip axis length (HAL) and women with trochanteric fracture had a significantly larger neck-shaft angle (NSA). Both groups had significantly lower cross-sectional moment of inertia (CSMI), and only women with neck fracture had a significantly higher buckling ratio (BR) compared to their respective controls. According to the multiple logistic regression analysis, women with neck fracture had a significantly longer HAL, lower CSMI, and 
higher BR, and women with trochanteric fracture had a significantly smaller cortical CSA of the femoral neck. We conclude that clinical CT may be useful for the assessment of the risk of neck and trochanteric fracture. 
Key words: osteoporosis, hip fracture, hip geometry, bone mineral density (BMD), computed tomography (CT) 


\section{INTRODUCTION}

The incidence of vertebral fracture increases linearly with aging and significantly correlates with bone mineral density (BMD). The incidence of hip fracture, on the other hand, rises exponentially with aging, suggesting that age-related factors other than BMD contribute greatly to the fragility of the proximal femur.

Three-dimensional (3D) analysis of the proximal femur is difficult, because of the complicated structure and bone density distribution in this region. Hip structure analysis (HSA) ${ }^{1)}$ is a clinically useful approach to assess BMD and geometry on the basis of dual X-ray absorptiometry (DXA) data, and biomechanical indices are derived from these geometrical parameters. In a large prospective population-based study with 147 incident hip fractures, baseline BMD and geometry of the proximal hip were analyzed by $\mathrm{HSA}^{2)}$, and it was found that hip fracture cases had lower BMD, thinner cortices, greater bone width, lower strength, and higher instability at baseline. However, there are several limitations to the HSA program; most of the geometrical parameters of HSA depend on assumptions about the shape of the cross-section and fixed percentages of cortical bone, and all of the geometrical parameters are derived from bone density. A recent study on DXA-based HSA in a large population-based cohort ${ }^{3)}$ concluded that HSA was prognostically equivalent, but not superior to BMD, in hip fracture prediction.

Volumetric quantitative computed tomography (QCT) has become an increasingly useful clinical research tool for analyzing hip geometry and measuring volumetric BMD ${ }^{4-7)}$. Recently, multi-detector row CT (MDCT) has been applied to obtain 3D isometric volumetric data to perform multiplanar reconstruction at an optimal location and to calculate the geometrical parameters ${ }^{8)}$. 3D CT data are advantageous to obtain cross-sectional images of the femoral neck (FN) as well as appropriate axes of the FN 
and femoral shaft. There are still some difficulties in the CT analysis of bone geometry, for example, in defining the threshold values for separating the cortex from the spongiosa, the need to avoid partial volume effects and the requirement of algorithms to contour the cortex of the voxel data.

The details of cross-sectional neck geometry have been investigated by means of cadaver or biopsy samples using high resolution (200 microns) peripheral QCT (HR-pQCT) ${ }^{9-11)}$. However, there have been only a few reports on the in vivo assessment of hip geometry in relation to fracture risk determination ${ }^{7)}$. Two case-control studies (neck and trochanteric fracture) were designed to investigate the contribution of the geometry and BMD of the proximal femur to bone strength in Japanese elderly women. Additionally we also investigated the potential application of clinical CT for the evaluation of hip BMD and geometry. 


\section{METHODS}

\section{Subjects}

Hip fracture cases included the women who experienced femoral neck or trochanteric fractures between 2007 and 2008 in Aichi and Nagasaki prefecture. One woman in the age range of \pm 5 years for each hip fracture case was randomly selected as a control subject from the 208 healthy volunteer women ages 51 to 87 . The selection was performed from the whole hip fracture case file without information on the type of fracture.

For the comparison of the characteristics of the hip geometry in the neck fracture and trochanteric fracture cases, two data sets were prepared. Subjects in the neck fracture study included 20 Japanese women with neck fracture (age: 68-86 years old, mean \pm SD; 80.1 \pm 4.5$)$ and age-matched control women (71-83 years old, 79.2 \pm 2.6$)$. As shown in Table 1 , the neck fracture subjects had a marginally lower body weight compared to their control subjects $(p=0.05)$. Subjects in the trochanteric fracture study included 16 Japanese women with trochanteric fracture (68-87 years old, 82.6 \pm 5.0$)$ and their age-matched controls (71-87 years old, 80.8 \pm 3.8 ). There were no significant difference in body weight/height between the trochanteric fracture and control subjects (Table 2). For the case subjects, CT examination of the proximal femur was performed between the date of admission and the date of surgery. The CT data on the control subjects were obtained in 2008. All the data were obtained at either the Nagasaki University Hospital or the National Center for Geriatrics and Gerontology Hospital.

The study was reviewed and approved by the Internal Review Boards at both Nagasaki University Hospital and the National Center for Geriatrics and Gerontology. Written informed consent to participate was obtained from all subjects. 


\section{CT data acquisition}

The MDCT scanners used were an Aquilion 16 (Toshiba Medical Systems Corporation, Tokyo, Japan) at Nagasaki University Hospital and a SOMATOM Cardiac 64 (Siemens, AG, Germany) at the National Center for Geriatrics and Gerontology Hospital. The reference phantom was a B-MAS200 (Fujirebio Inc, Japan) containing hydroxyapatite at $0,50,100,150$ and $200 \mathrm{mg} / \mathrm{cm}^{3}$. The scanning conditions were adjusted to $120 \mathrm{kV}, 250 \mathrm{~mA}$ and a reconstruction thickness of $0.5 \mathrm{~mm}$, and the spatial resolution was $0.625 \times 0.625 \mathrm{~mm}$ at Nagasaki University Hospital and $0.652 \times 0.652 \mathrm{~mm}$ at the National Center for Geriatrics and Gerontology Hospital. The radiation dose was 19.7mGy at maximal, as shown by the CTDIvol.

Both CT scanners displayed good linear correlation between the CT values and HA concentrations $(\mathrm{r}=0.9999, \mathrm{p}<0.0001)$. The individual rods containing a different density of HA exhibited good stability (\%CV=0.01-0.3\%). For cross-calibration between the Toshiba and Siemens scanners, a QCT-PRO QA phantom, containing $\mathrm{K}_{2} \mathrm{HPO}_{4}$ equivalent density range from $-50 \mathrm{mg} / \mathrm{cc}$ to $375 \mathrm{mg} / \mathrm{cc}$, was used. The linear correlation $(y=-6.4+1.03 x(r=0.9998, p<0.0001))$ was considered sufficient to pool all data from the two institutes together.

\section{Analysis of BMD and geometry obtained by CT}

BMD as well as bone geometry of the proximal femur was analyzed by M.I using commercial software (QCT PRO; Mindways Software, San Francisco, CA, USA). BMD, bone mass, the bone area as well as hip axis length (HAL), the femoral neck angle (FNA) and neck width were calculated. FNA was defined as the angle between 
the axis of the FN and the axis of a cross-section of shaft, and the neck shaft angle (NSA) was calculated as FNA plus 90 degrees. In the cross-sectional FN, BMD and the cross-sectional area (CSA) of the total/cortical/trabecular regions, and cortical thickness, cortical perimeter, curvature (reciprocal of radius of curvature), and distance to the center of the bone mass (distance to CM) were obtained (Fig. 1). Fig. 1 (E) indicates 16 sector points and lines on the basis of the center of the mass to calculate the distance to CM. As biomechanical parameters, the cross-sectional moment of inertia (CSMI) depending on the principle axis (Fig.1) and the buckling ratio (BR) were calculated from these BMD and geometry data. BR was calculated as the distance to CM divided by the average cortical thickness in this study. The reproducibility (\% coefficient of variation) of the analysis by the QCT PRO program was calculated using five repeated analyses with visual matching each time from ten healthy subject CT data sets without visible artifact; total BMD 1.62\%, cortical BMD 5.17\%, trabecular BMD 1.57\%, total mass $2.73 \%$, total area $3.53 \%$, cortical area $0.83 \%$, trabecular area $4.67 \%$, FN width 1.79\%, HAL 2.11\%, NSA 5.70\%, cortical perimeter 1.61\%, cortical thickness 7.25\%, and distance to CM 2.02\%.

\section{Statistical analysis}

Data were analyzed using the Statistical Analysis System Version 9.1.3 (SAS Institute Inc. Cary, NC). The student t-test was used to determine the significance of differences between fracture cases and controls. A logistic regression model was used to explore the simultaneous effects of bone parameters on fractures. Starting with a full model, including variables that showed a p-value of less than 1.0 in the univatiate analysis, the most appropriate model was selected on the basis of Akaike's information 
criteria (AIC). The odds ratio (OR) and the 95\% confidence interval (CI) were calculated for each covariate included in the model. 


\section{RESULTS}

Table 1 shows the univariate comparisons of variables between the femoral neck fracture cases and the controls. Case subjects had significantly lower total and cortical BMD, larger total and trabecular CSA, smaller cortical CSA, smaller cortical thickness, shorter distance to CM, lower CSMI, and higher BR, compared to the control subjects. HAL was marginally longer in the case subjects compared to the control subjects, while NSA did not significantly differ between the cases and controls.

Table 2 shows the univariate comparisons of variables between the trochanteric fracture cases and their controls. Case subjects had significantly lower total and cortical BMD, larger NSA, larger trabecular CSA, smaller cortical CSA, smaller cortical thickness, shorter cortical perimeter, shorter distance to CM, and lower CSMI, compared to the control subjects. There was no significant difference in HAL or BR between the cases and controls.

Tables 3 and 4 show the results of multiple logistic regression analysis for neck fracture and trochanteric fracture. For neck fracture, a higher BR was significantly associated with risk of fracture. Longer HAL and smaller CSMI were marginally associated with risk of neck fracture (Table 3). For trochanteric fracture, smaller cortical CSA was significantly associated with risk of fracture (Table 4). NSA was not significantly associated with risk of trochanteric fracture. 


\section{DISCUSSION}

Since it is generally recognized that, compared to neck fracture, trochanteric fracture occurs in subjects with a lower BMD and in more elderly subjects, it is preferable to analyze the neck fracture and trochanteric fracture, separately. We designed a study to investigate the characteristic geometrical factors predisposing to neck and trochanteric fracture on the basis of independent two case-control studies, and the geometry and BMD of the proximal femur were analyzed using clinical CT.

According to the multiple logistic regression analysis, a greater BR, an approximate index of cortical instability, was associated with neck fracture. A lower CSMI, a classical biomechanical parameter of bending stress, and a longer HAL were marginally associated with neck fracture. These biomechanical findings indicate that neck fracture is predisposed to both cortical instability and bending load. Upon falling, a femoral neck with a longer HAL is considered to tend to be provoked into local buckling due to the bending load.

The usefulness of BR in the prediction of hip fracture using DXA-based HSA has been controversial ${ }^{2-3)}$. In the present CT-based HSA study, BR was calculated as the distance to the CM divided by the average cortical thickness. The biomechanical parameter of BR was significant in relation to neck fracture (OR=2.56 (95\%CI 1.21, 5.43), $\mathrm{p}<0.01$ ), indicating that buckling leads to neck fracture when these two geometrical indices interact. As it is well known that buckling occurs in long bones having a thin wall, a thin cortex may be involved in the risk for neck fracture. Since it is difficult to measure cortical thickness (which will be discussed later), the measure of cortical thickness might have a lower power of prediction in the risk of hip fracture. BR has been proved useful in predicting the risk of fracture, and it includes two important 
geometrical indices related to bone strength.

The multiple logistic regression analysis demonstrated that the cortical CSA was significantly correlated with trochanteric fracture. Cortical CSA has been demonstrated to be an estimator of compression strength ${ }^{3)}$. Trochanteric fracture may be more closely associated with a weakness for compression load, while neck fracture more with a weakness for bending load

In both neck and trochanteric fracture, the cortical and total BMD values were significantly different from the respective values in control women, while these BMD values were not selected in the multiple logistic regression analysis. This indicates that BMD values have no strong influence on neck fracture or trochanteric fracture after taking various geometrical parameters into consideration. The results of comparisons between groups showed that the t-values of the BR in neck fracture was greater than that of total BMD (see Table 1), and that the t-value of cortical CSA was similar to that of total BMD (see Table 2), which may in part explain these findings.

Among the geometrical parameters, HAL $^{12-14)}$ and NSA ${ }^{15-17)}$ have been under investigation for years in relation to hip fracture, using radiographs and DXA data. It is difficult to maintain a good positioning of the hip joint so as to be able to analyze the geometry using such techniques as HAL and NSA in the radiographs and DXA after hip fracture, since the legs are usually abducted and outwardly rotated ${ }^{18)}$. If the measurement is performed after an operation, the parameters are influenced by the effect of limb disuse. In this respect, CT scanning has the advantage of not being influenced by leg positioning ${ }^{19)}$. Three-dimensional CT evaluation is capable of high reproducibility in assessing the efficacy of anti-osteoporotic agents on the individual trabecular and cortical BMD, as well as making a precise measurement of geometrical 
properties.

Another advantage of CT examination is the capacity to obtain FN cross-sectional images, including both cortical thickness in local regions and distance to CM. Ex vivo study demonstrated that the local difference in cortical thickness and density in the cross-sectional FN was important in terms of the biomechanical properties of the proximal femur ${ }^{9)}$. The DXA-based HSA also provides the distance to CM based on projectional data, and a measurement of biomechanical parameters on the basis of its architectural distribution from 2D images, however, it might be useful as a complementary or alternative approach to provide relevant information not evident in the volume-averaged BMD measurement.

The analysis of thin cortex using clinical CT is challenging; the voxel size is not small enough to define such a thin cortical contour and hence cannot measure the cortical thickness. Because of a partial volume effect, the decrease in cortical BMD tends to be overestimated. In the present study using clinical CT, a few cases with extremely thin cortex exhibited "bone defects" (i.e. areas with zero bone density) in the posterior region of FN. We calculated these defects as a thickness of "zero", and therefore may have overestimated the reduction in cortical thickness.

We conclude that clinical CT may be a useful tool for the assessment of the risk of neck and trochanteric fracture. Women with neck fracture had a higher BR, longer HAL and lower CSMI, while women with trochanteric fracture had a smaller cortical cross-sectional area of the femoral neck. Since our sample size was small, larger studies are needed to ultimately determine the relationship of hip geometry using clinical CT to hip fracture risk. 


\section{Acknowledgements}

The authors thank Tomoko Nakata, Toru Fukuda and Takako Shimogama (Division of Radiology, Nagasaki University Hospital), and Yoshimasa Yoshino and Hiroyuki Fujisawa (Department of Radiology, National Center for Geriatrics and Gerontology) for technical assistance, and Dr Kyoji Ikeda (Department of Bone and Joint Disease, National Center for Geriatrics and Gerontology, Obu, Aichi, Japan;) for comments on the manuscript. This study was supported in part by a grant for the Promotion of Fundamental Studies in Health Sciences of the National Institute of Biomedical Innovation (NIBIO) of Japan (MF-14 and 06-31 to MI), and by a grant from Elli Lilly company (to MI). Pacific Edit reviewed the manuscript before submission. 


\section{References}

1) Beck TJ, Ruff CB, Warden KE, Scott WW Jr, Rao GU. Predicting femoral neck strength from bone mineral data. A structural approach. Invest Radiol 1990; 25:6-18.

2) Rivadeneira F, Zillikens MC, De Laet CEDH, Hofman A, Uitterlinden AG, Beck TJ, Pols1 HAP. Femoral neck BMD is a strong predictor of hip fracture susceptibility in elderly men and women because it detects cortical bone instability: The Rotterdam Study. J Bone Miner Res 2007; 22:1781-1790.

3) Kaptoge S, Beck TJ, Reeve J, Stone KL, Hillier TA, Cauley JA, Cummings SR. Prediction of indicent hip fracture risk by femur geometry variables measured by hip structural analysis in the study of osteoporotic fractures. J Bone Mier Res 2008; 23: 1892-1904.

4) Black DM, Bilezikian JP, Ensrud KE, Greenspan SL, Palermo L, Hue T, et al. One year of alendronate after one year of parathyroid hormone (1-84) for osteoporosis. N Engl J Med 2005; 353: 555-565.

5) Black DM, Greenspan SL, Ensrud KE, Palermo L, McGowan JA, Lang TF, et al. The effects of parathyroid hormone and alendronate alone or in combination in postmenopausal osteoporosis. $\mathrm{N}$ Engl J Med 2003; 349: 1207-1215.

6) Lang T, LeBlanc A, Evans H, Lu Y, Genant H, Yu A. Cortical and trabecular bone mineral loss from the spine and hip in long-duration spaceflight. J Bone Miner Res 2004; 19: 1006-1012.

7) Riggs BL, Melton LJ III, Robb RA, Camp JJ, Atkinson EJ, Peterson JM, et al. Population-based study of age and sex differences in bone volumetric density, size, geometry, and structure at different skeletal sites, J Bone Miner Res 2004; 19: 1945-1954.

8) Ito M, Ikeda K, Nishiguchi M, Shindo H, Uetani M, Hosoi T, Orimo H. Multi-detector row CT imaging of vertebral microstructure for evaluation of fracture risk. J Bone Miner Res 2005; 20 : 1828-1836.

9) Mayhew PM, Thomas CD, Clement JG, Loveridge N, Beck TJ, Bonfield W, et al. Relation between age, femoral neck cortical stability, and hip fracture risk. Lancet 2005; 366: 129-135.

10) Crabtree N, Loveridge N, Parker M, Rushton N, Power J, Bell KI, Beck TJ. Intracapsular hip fracture and the region-specific loss of cortical bone: Analysis by peripheral quantitative computed tomography. J Bone Miner Res 2001; 16: 1318-1328.

11) Power J, Loveridge N, Lyon A, Rushton N, Parker M, Reeve J. Bone remodeling the endocortical surface of the human femoral neck: A mechanism for regional cortical thinning in cases of hip fracture. J Bone Miner Res 2003; 18: 1775-1780.

12) Nakamura T, Turner CH, Yoshikawa T, Slemenda CW, Peacock M, Burr DB et al. Do variations in hip geometry explain differences in hip fracture risk between Japanese and white Americans? 
J Bone Miner Res 1994; 9 :1071-1076.

13) Greedale GA. Young JT, Huang M-H, Bucur A, Wang Y, Seeman T. Hip axis length in mid-life Japanese and Caucasian U.S. residents: no evidence for and ethnic difference. Osteoporosis Int 2003; 14: 320-325.

14) Cummings SR, Cauley JA, Palermo L, Ross PD, Wasnich RD, Black D, Faulkner KG for the study of osteoporotic fracture research garup. Racial differences in hip axis lengths might explain racial differences in rates of hip fractue. Osteoporosis Int 1994; 4: 226-229.

15) Gnudi S, Malavolta N, Testi D, Viceconti M. Differences in proximal femur geometry distinguish vertebral from femoral neck fractures in osteoporotic women. Br J Radiol 2004; 77: 219-223.

16) Gnudi S, Ripamonti C, Gualtieri G, Malavolta N. Geometry of proximal femur in the prediction of hip fracture in osteoporotic women. Br J Radiol 1999; 72: 729-733.

17) Alonso CG, Curiel MD, Carranza FH, Cano RP, Perez AD, the Multicenter Project for Research in Osteoporosis. Femoral bone mineral density, neck-shaft angle and mean femoral neck width as predictors of hip fracture in men and women. Osteoporos Int 2000; 11: 714-720.

18) Michelotti J, Clark J. Femoral neck length and hip fracture risk. J Bone Miner Res 1999; 14:1714-1720.

19) Pulkkinen P, Eckstein F, Lochmuller E-M, Kuhn V, Jamsa T. Association of geometric factors and failure load level with the distribution of cervical vs. trochanteric hip fractures. J Bone Miner Res 2006; 21: 895-901. 
Figure legend

Figure 1

The measurements of the cross-sectional femoral neck (FN)

(A) Surface rendering CT images to define the longitudinal axis of the femoral neck and region of interest for cross-sectional FN

(B) Cortical bone image

(C) Principle axes

(D) Center of bone mass

(E) Sector points and lines 
Figure1

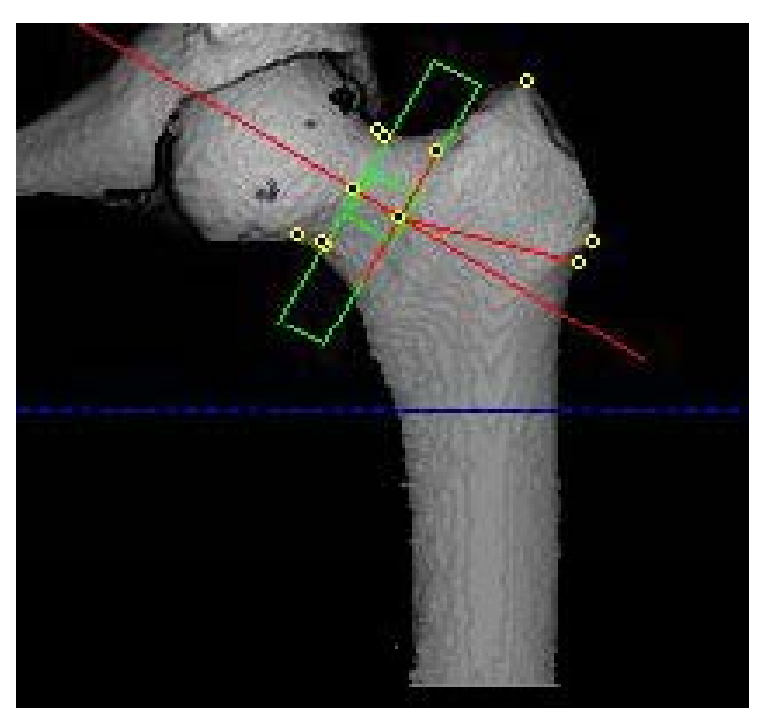

(A)

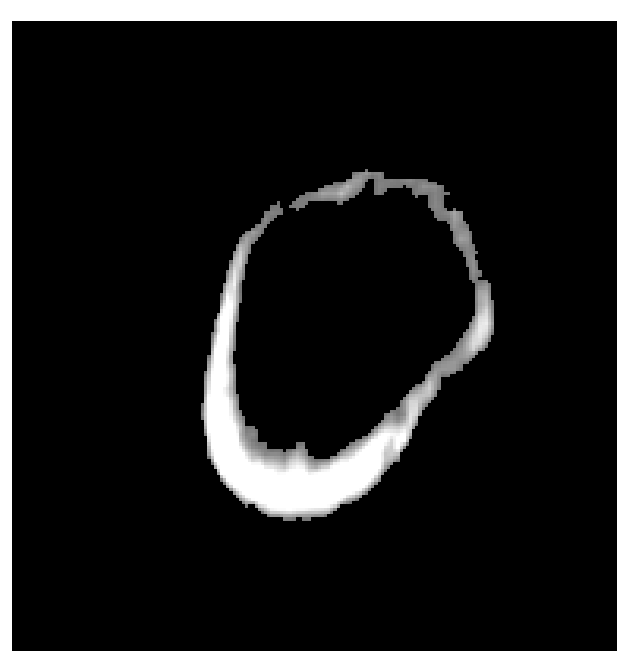

(B)

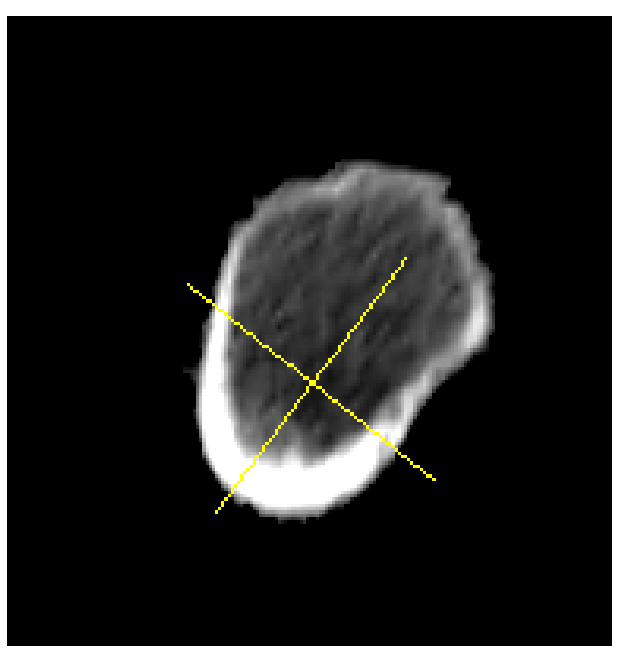

(C)

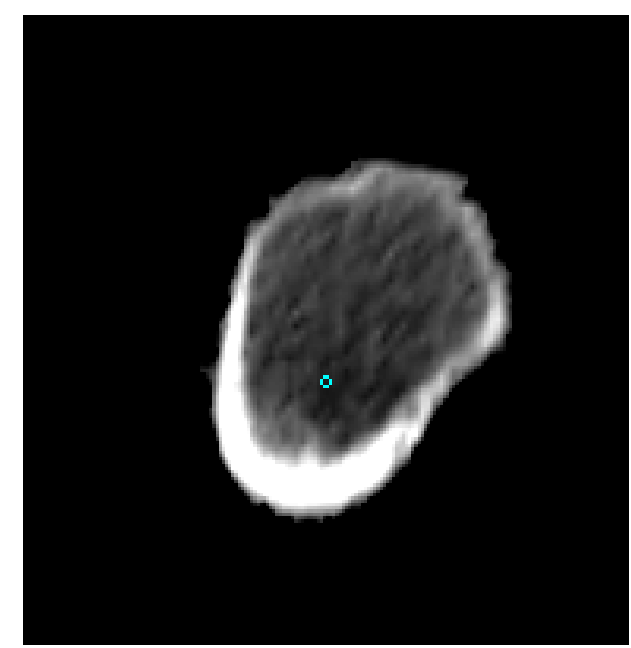

(D)

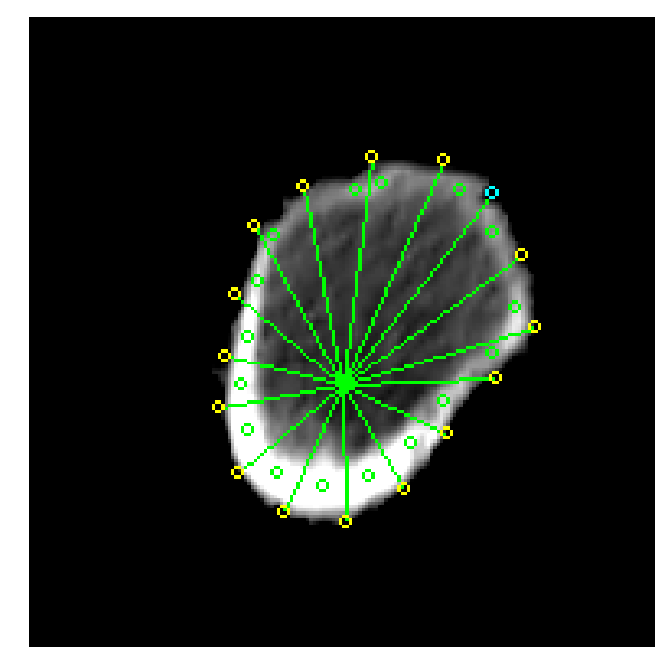

(E) 
Table 1. Comparison of variables between neck fracture cases and controls.

\begin{tabular}{|c|c|c|c|c|}
\hline & case $(n=20)$ & control $(n=20)$ & t-value & $\mathrm{p}$-value \\
\hline Age (years) & $80.1 \pm 4.5$ & $79.2 \pm 2.6$ & 0.73 & 0.47 \\
\hline Weight (kg) & $42.3 \pm 8.9$ & $47.5 \pm 7.2$ & 2.05 & 0.05 \\
\hline Height (cm) & $145.6 \pm 7.2$ & $146.1 \pm 6.2$ & 0.24 & 0.81 \\
\hline Total BMD $\left(\mathrm{g} / \mathrm{cm}^{2}\right)$ & $0.416 \pm 0.077$ & $0.520 \pm 0.100$ & 3.69 & $<0.001$ \\
\hline Cortical BMD $\left(\mathrm{g} / \mathrm{cm}^{2}\right)$ & $0.288 \pm 0.068$ & $0.369 \pm 0.111$ & 2.80 & 0.009 \\
\hline Trabecular BMD $\left(\mathrm{g} / \mathrm{cm}^{2}\right)$ & $0.133 \pm 0.042$ & $0.141 \pm 0.046$ & 0.57 & 0.57 \\
\hline Neck-shaft angle $\left(^{\circ}\right.$ ) & $129.2 \pm 5.3$ & $128.7 \pm 4.5$ & 0.31 & 0.76 \\
\hline Neck width (mm) & $25.7 \pm 2.6$ & $25.2 \pm 2.8$ & 0.64 & 0.53 \\
\hline Hip Axis Length (mm) & $107.7 \pm 8.5$ & $103.2 \pm 4.6$ & 2.05 & 0.05 \\
\hline Total CSA $\left(\mathrm{cm}^{2}\right)$ & $7.02 \pm 0.82$ & $6.53 \pm 0.64$ & 2.14 & 0.04 \\
\hline Cortical CSA $\left(\mathrm{cm}^{2}\right)$ & $0.85 \pm 0.28$ & $1.12 \pm 0.35$ & 2.70 & 0.01 \\
\hline Trabecular CSA $\left(\mathrm{cm}^{2}\right)$ & $6.05 \pm 0.77$ & $5.40 \pm 0.89$ & 2.46 & 0.02 \\
\hline Cortical thickness (mm) & $1.23 \pm 0.43$ & $1.58 \pm 0.42$ & 2.62 & 0.01 \\
\hline Cortical perimeter (mm) & $5.35 \pm 0.80$ & $5.72 \pm 0.76$ & 1.51 & 0.14 \\
\hline Distance to CM (mm) & $10.98 \pm 2.32$ & $12.49 \pm 1.88$ & 2.27 & 0.03 \\
\hline Curvature $(1 / \mathrm{cm})$ & $0.64 \pm 0.13$ & $0.70 \pm 0.09$ & 1.81 & 0.08 \\
\hline $\operatorname{CSMI}\left(\mathrm{cm}^{4}\right)$ & $0.32 \pm 0.10$ & $0.54 \pm 0.23$ & 3.81 & $<0.001$ \\
\hline BR & $12.22 \pm 1.69$ & $8.34 \pm 2.13$ & 6.38 & $<0.001$ \\
\hline
\end{tabular}

Values are means \pm standard deviation.

BMD: bone mineral density, CSA: cross-sectional area, CM: center of bone mass, CSMI: cross-sectional moment of inertia, BR: buckling ratio 
Table 2. Comparison of variables between trochanteric fracture cases and controls.

\begin{tabular}{|c|c|c|c|c|}
\hline & case $(n=16)$ & control $(n=16)$ & t-value & $\mathrm{p}$-value \\
\hline Age (years) & $82.6 \pm 5.0$ & $80.8 \pm 3.8$ & 1.10 & 0.28 \\
\hline Weight (kg) & $43.0 \pm 8.3$ & $45.8 \pm 6.4$ & 1.04 & 0.30 \\
\hline Height (cm) & $147.0 \pm 5.0$ & $144.4 \pm 6.8$ & 1.20 & 0.24 \\
\hline Total BMD $\left(\mathrm{g} / \mathrm{cm}^{2}\right)$ & $0.382 \pm 0.084$ & $0.501 \pm 0.081$ & 4.04 & $<0.001$ \\
\hline Cortical BMD $\left(\mathrm{g} / \mathrm{cm}^{2}\right)$ & $0.247 \pm 0.075$ & $0.349 \pm 0.094$ & 3.40 & 0.002 \\
\hline Trabecular BMD $\left(\mathrm{g} / \mathrm{cm}^{2}\right)$ & $0.130 \pm 0.042$ & $0.147 \pm 0.034$ & 1.24 & 0.22 \\
\hline Neck-shaft angle $\left({ }^{\circ}\right.$ ) & $133.2 \pm 6.3$ & $128.0 \pm 4.5$ & 2.67 & 0.01 \\
\hline Neck width (mm) & $25.3 \pm 2.2$ & $26.8 \pm 2.9$ & 1.63 & 0.11 \\
\hline Hip Axis Length (mm) & $105.4 \pm 7.4$ & $103.6 \pm 6.4$ & 0.75 & 0.46 \\
\hline Total CSA $\left(\mathrm{cm}^{2}\right)$ & $6.78 \pm 0.85$ & $6.96 \pm 0.84$ & 0.61 & 0.55 \\
\hline Cortical CSA $\left(\mathrm{cm}^{2}\right)$ & $0.65 \pm 0.34$ & $1.11 \pm 0.33$ & 3.92 & $<0.001$ \\
\hline Trabecular CSA $\left(\mathrm{cm}^{2}\right)$ & $6.56 \pm 0.92$ & $5.85 \pm 0.90$ & 2.24 & 0.03 \\
\hline Cortical thickness (mm) & $1.13 \pm 0.31$ & $1.36 \pm 0.29$ & 2.16 & 0.04 \\
\hline Cortical perimeter (mm) & $5.01 \pm 0.94$ & $5.89 \pm 0.82$ & 2.81 & 0.009 \\
\hline Distance to CM (mm) & $10.43 \pm 2.39$ & $12.63 \pm 1.68$ & 3.02 & 0.005 \\
\hline Curvature $(1 / \mathrm{cm})$ & $0.62 \pm 0.14$ & $0.66 \pm 0.05$ & 1.09 & 0.29 \\
\hline $\operatorname{CSMI}\left(\mathrm{cm}^{4}\right)$ & $0.36 \pm 0.12$ & $0.55 \pm 0.23$ & 2.89 & 0.008 \\
\hline BR & $9.27 \pm 1.40$ & $9.53 \pm 1.71$ & 0.47 & 0.64 \\
\hline
\end{tabular}

Values are means \pm standard deviation.

BMD: bone mineral density, CSA: cross-sectional area, CM: center of bone mass, CSMI: cross-sectional moment of inertia, BR: buckling ratio 
Table 3. Multiple logistic regression analysis for neck fracture.

\begin{tabular}{lcccc}
\hline variables & unit & OR & $95 \%$ CI & p-value \\
\hline Hip Axis Length $(\mathrm{mm})$ & 5 & 2.15 & $0.95,4.87$ & 0.07 \\
CSMI $\left(\mathrm{cm}^{4}\right)$ & -0.05 & 1.52 & $0.99,2.34$ & 0.06 \\
BR & 1 & 2.56 & $1.21,5.43$ & 0.01 \\
\hline
\end{tabular}

CSMI: cross-sectional moment of inertia, BR: buckling ratio 
Table 4. Multiple logistic regression analysis for trochanteric fracture.

\begin{tabular}{lcccc}
\hline variables & unit & OR & $95 \%$ CI & p-value \\
\hline Neck-shaft angle $\left(^{\circ}\right)$ & 5 & 2.15 & $0.84,5.47$ & 0.11 \\
Cortical CSA $\left(\mathrm{cm}^{2}\right)$ & -0.1 & 1.47 & $1.08,1.99$ & 0.01 \\
\hline
\end{tabular}

CSA: cross-sectional area 